\title{
Multiple Types of Cerebellar Target Neurons and Their Circuitry in the Vestibulo-ocular Reflex
}

\author{
Minyoung Shin, ${ }^{1}$ Setareh H. Moghadam, ${ }^{1}$ Chris Sekirnjak, ${ }^{1,2}$ Martha W. Bagnall, ${ }^{1,2}$ Kristine E. Kolkman, ${ }^{1,2}$ \\ Richard Jacobs, ${ }^{1}$ Michael Faulstich, ${ }^{1}$ and Sascha du Lac ${ }^{1,2}$ \\ ${ }^{1}$ Salk Institute for Biological Studies and Howard Hughes Medical Institute, La Jolla, California 92037, and 2Neurosciences Graduate Program, University of \\ California, San Diego, La Jolla, California 92093
}

The cerebellum influences behavior and cognition exclusively via Purkinje cell synapses onto neurons in the deep cerebellar and vestibular nuclei. In contrast with the rich information available about the organization of the cerebellar cortex and its synaptic inputs, relatively little is known about microcircuitry postsynaptic to Purkinje cells. Here we examined the cell types and microcircuits through which Purkinje cells influence an oculomotor behavior controlled by the cerebellum, the horizontal vestibulo-ocular reflex, which involves only two eye muscles. Using a combination of anatomical tracing and electrophysiological recordings in transgenic mouse lines, we identified several classes of neurons in the medial vestibular nucleus that receive Purkinje cell synapses from the cerebellar flocculus. Glycinergic and glutamatergic flocculus target neurons (FTNs) with somata densely surrounded by Purkinje cell terminals projected axons to the ipsilateral abducens and oculomotor nuclei, respectively. Of three additional types of FTNs that were sparsely innervated by Purkinje cells, glutamatergic and glycinergic neurons projected to the contralateral and ipsilateral abducens, respectively, and GABAergic neurons projected to contralateral vestibular nuclei. Densely innervated FTNs had high spontaneous firing rates and pronounced postinhibitory rebound firing, and were physiologically homogeneous, whereas the intrinsic excitability of sparsely innervated FTNs varied widely. Heterogeneity in the molecular expression, physiological properties, and postsynaptic targets of FTNs implies that Purkinje cell activity influences the neural control of eye movements in several distinct ways. These results indicate that the cerebellum regulates a simple reflex behavior via at least five different cell types that are postsynaptic to Purkinje cells.

\section{Introduction}

The cerebellum plays crucial roles in the integration of sensory and motor signals, the coordination of movement and cognition, and motor learning. Purkinje cells in specifically arranged zones in the cerebellum receive sensory and/or motor signals via widespread mossy fibers and climbing fibers from well defined subregions of the inferior olive (for review, see Ruigrok, 2010) and provide the sole output of the cerebellar cortex. Despite a wealth of information on the organization of signal processing within the cerebellar cortex (Voogd and Glickstein, 1998; Apps and Garwicz, 2005), relatively little is known about how Purkinje cell target neurons in the cerebellar and vestibular nuclei control individual behaviors.

The cerebellar and vestibular nuclei have received increasing attention as potential sites of plasticity that are critical for cerebellar motor learning (Mauk et al., 1998; Medina et al., 2000;

Received Feb. 11, 2011; revised April 25, 2011; accepted May 2, 2011.

Author contributions: M.S., C.S., M.W.B., K.K., R.J., M.F., and S.d.L. designed research; M.S., S.H.M., C.S., M.W.B., K.K., R.J., and M.F. performed research; M.S., C.S., M.W.B., K.K., R.J., M.F., and S.d.L. analyzed data; M.S., C.S., M.W.B., and S.d.L. wrote the paper.

This work was funded by NIH Grant EY-11027 and the Howard Hughes Medical Institute. M.B. was funded by a National Science Foundation graduate research fellowship. We thank Susanna Fenstermacher for technical assistance.

Correspondence should be addressed to Sascha du Lac, Salk Institute for Biological Studies and Howard Hughes Medical Institute, 10010 N. Torrey Pines Road, La Jolla, CA 92037 . E-mail: sascha@salk.edu.

DOI:10.1523/JNEUROSCI.0768-11.2011

Copyright $\odot 2011$ the authors $\quad 0270-6474 / 11 / 3110776-11 \$ 15.00 / 0$
Kassardjian et al., 2005; Shutoh et al., 2006), and candidate cellular mechanisms of both intrinsic and synaptic plasticity in cerebellar and vestibular nuclei have been identified (Aizenman and Linden, 2000; Nelson et al., 2005; Gittis and du Lac, 2006; Pugh and Raman, 2006; Zhang and Linden, 2006; McElvain et al., 2010; Person and Raman, 2010). Establishing secure links between such cellular mechanisms of plasticity and their behavioral consequences for cerebellar learning requires knowledge about how microcircuits postsynaptic to Purkinje cells influence motor performance. Most behaviors that serve as models for cerebellar learning, however, are mediated by complex circuits in which several synapses are interposed between Purkinje cells and downstream motor neurons.

One of the simplest behaviors regulated by the cerebellum is the vestibulo-ocular reflex (VOR), which in the horizontal plane is controlled by only two extraocular muscles. The VOR functions to stabilize images on the retina during self-motion; its excellent performance throughout life is maintained by robust, experience-dependent learning, which relies on the cerebellum (Marr, 1969; Albus, 1971; Ito, 1972; De Zeeuw et al., 1998; Shutoh et al., 2003; for review, see De Zeeuw and Yeo, 2005; Highstein and Holstein, 2006). Motor learning in the VOR depends on Purkinje cells in the floccular lobe of the cerebellum which, in turn, synapse onto "flocculus target neurons" (FTNs) in the vestibular nuclei, some of which are thought to directly drive ocular motoneurons (Ito et al., 1977; Stahl and Simpson, 1995). Recordings from FTNs in conjunction with motor learning in the VOR 
have revealed pronounced changes in their firing responses during head and eye movements (Lisberger et al., 1994a; Partsalis et al., 1995), indicating that plasticity in FTNs and/or synapses onto them contributes to cerebellar learning. Robust motor learning, together with tractable circuitry interposed between the cerebellum and motor neurons, makes the VOR an excellent model for investigating the microcircuitry by which the cerebellum influences behavior. This study uses transgenic mice expressing fluorescent proteins under specific promoters together with retrograde labeling and assessment of intrinsic physiological excitability to define the cell types and axonal projections that mediate cerebellar floccular influence over the horizontal VOR.

\section{Materials and Methods}

Animals. Mice of both genders from the following lines were used for electrophysiology experiments and histology experiments: L7-tau-GFP mice expressing GFP under the Purkinje cell-specific L7 promoter (Sekirnjak et al., 2003); GlyT2 mice expressing GFP under glycine transporter 2 promoter (Zeilhofer et al., 2005); YFP-16 mice expressing YFP (yellow fluorescent protein) under thyl promoter, which labels both glycinergic and glutamatergic neurons in vestibular nuclei (Feng et al., 2000; Bagnall et al., 2007); GIN mice expressing GFP under the glutamic acid decarboxylase (GAD65) promoter, which labels a subset of GABAergic neurons (Oliva et al., 2000; Bagnall et al., 2007); GAD65 mice expressing GFP under the GAD65 promoter in a subset of GABAergic neurons different from that of GIN mice (López-Bendito et al., 2004); and GAD67-GFP knock-in mice (Tamamaki et al., 2003). All mice were housed with a $12 \mathrm{~h} \mathrm{light/dark} \mathrm{cycle} \mathrm{and} \mathrm{ad} \mathrm{libitum} \mathrm{water} \mathrm{and} \mathrm{food} \mathrm{access.}$ All experiments were performed under the approval of the Salk Institute Animal Care and Use Committee rules.

Tracer injection. For immunohistochemical studies, the abducens nucleus, the oculomotor nucleus (OMN), or the medial vestibular nucleus $(\mathrm{MVN})$ of adult mice from each mouse line was targeted stereotaxically. Animals were anesthetized with isoflurane inhalation and immobilized in the stereotaxic device with earbars. After exposure of the skull, a hole was drilled $\sim 6 \mathrm{~mm}$ posterior from bregma. For abducens injection, crystals of tetramethylrhodamine dextran (Invitrogen) or biotin-conjugated dextran (BDA) (Invitrogen) were dissolved in 0.1 M PBS (pH 7.4) with final a concentration of $0.1 \mathrm{~g} / \mathrm{ml}$. Borosilicate glass micropipettes (World Precision Instruments) for delivery of dextran tracers were prepared with a tip diameter of $20 \mu \mathrm{m}$ using a P-97 puller (Sutter Instruments). The abducens nucleus of the left hemisphere was targeted according to the stereotaxic coordinates [in $\mathrm{mm}$ : $\mathrm{ML},-0.33$; anteroposterior (AP), -5.80 ; DV, -4.51 from bregma), and $3-5$ pulses $(20 \mathrm{~ms}$ at $1 \mathrm{~Hz})$ of compressed nitrogen (20 psi) were delivered to the injector to expel the dextran. For MVN injection, crystals of BDA were dissolved in $0.1 \mathrm{M}$ PBS. Borosilicate glass micropipettes were prepared with a tip diameter of 40 $\mu \mathrm{m}$. The MVN of the left hemisphere was targeted according to the stereotaxic coordinates (in mm: ML, -0.88 ; AP, $-6.00 ; \mathrm{DV},-4.00$ from bregma), and $5-10$ pulses $(20 \mathrm{~ms}$ at $1 \mathrm{~Hz}$ ) of compressed nitrogen (20 psi) were delivered to the injector to expel the whole dextran. For visualization of oculomotor nucleus-projecting neurons, solid crystals of tetramethylrhodamine dextran were microinjected following the protocol described previously (Sekirnjak and du Lac, 2006). Animals injected with tetramethylrhodamine dextran were killed $24-48 \mathrm{~h}$ after injection, and animals injected with BDA were killed 5-6 d after injection.

Immunohistochemistry. Mice were deeply anesthetized with Nembutal and transcardially transfused with $0.1 \mathrm{~m}$ PBS, followed by $4 \%$ paraformaldehyde (PFA) in 0.1 M PBS, pH 7.4. Brains were removed and further fixed in 4\% PFA for $1 \mathrm{~h}$ at room temperature (RT), and then placed in $30 \%$ sucrose in $\mathrm{PBS}$ overnight at $4^{\circ} \mathrm{C}$. Frozen brains were sectioned as $30 \mu \mathrm{m}$ free-floating sections in the coronal plane on a Microm sliding microtome (Mikron Instruments). For visualization of BDA-labeled neurons, free-floating sections were rinsed with $0.1 \mathrm{M}$ PBS, then permeabilized with $0.3 \%$ Triton X-100 in PBS (PBS-T) for $20 \mathrm{~min}$ at RT. Fluorescence-conjugated streptavidin solution (Invitrogen) was prepared at a final concentration of $10 \mu \mathrm{g} / \mathrm{ml}$ in PBS-T, and tissue sections were incubated in streptavidin solution for $30 \mathrm{~min}$ at RT.

Purkinje cells were labeled with mouse $\alpha$-calbindin (1:200, Abcam) by incubating for $2 \mathrm{~h}$ at RT, and visualized with $\alpha$-mouse-Cy3 (1:250; Sigma). Finally, free-floating sections were mounted on a glass slide and coverslipped with antifade gel containing $2.5 \%$ DABCO (1,2-diazabicyclo[2,2,2] octane).

For analysis of Purkinje cell terminals onto FTNs in conjunction with measurement of electrophysiological properties, neurons were filled with $0.1 \%(\mathrm{w} / \mathrm{v})$ biocytin (Sigma) during patch-clamp recording. Tissues were fixed in 4\% PFA in $0.1 \mathrm{M}$ PBS for $1 \mathrm{~h}$ at RT, and then in $30 \%$ sucrose in 0.1 м PBS overnight at $4^{\circ} \mathrm{C}$. Biocytin-filled neurons were visualized with Alexa Fluor 594-conjugated streptavidin $(10 \mu \mathrm{g} / \mathrm{ml})$ and mounted as described above.

Fluorescence microscopy and data analysis. Fluorescent images were taken using an Olympus BX60 light microscope equipped with a Hamamatsu CCD camera with a $10 \times$ [numerical aperture (NA) 0.3 ] or $40 \times$ (NA 1.0) objective. Images were collected digitally, and data analysis including cell counting was performed using NIH ImageJ software.

Locations and numbers of Purkinje axon terminals onto FTNs were analyzed using $z$-stack images taken with an Olympus FV300 confocal microscope equipped with a $60 \times$ objective, with an additional $2 \times$ or $3 \times$ digital zoom. Series of confocal images with $0.4 \mu \mathrm{m}$ interval were taken and reconstructed as $z$-stack images using ImageJ software. Purkinje cell axon terminals on the soma and proximal dendrites (up to $\sim 20 \mu \mathrm{m}$ from the soma) were counted manually.

Electron microscopy. Mice were anesthetized with Nembutal and transcardially perfused with PBS followed by $4 \%$ formaldehyde, $0.02 \%$ glutaraldehyde in PBS. The brain was removed and left in fixative until slicing. The brain was rinsed in ice-cold PBS, and the cerebellum and brainstem were cut into $50 \mu \mathrm{m}$ slices on a vibratome. Slices were placed in a chamber and imaged on a confocal microscope to locate GFP-labeled Purkinje cell terminals in the vestibular nucleus. Following imaging, slices were fixed in $2 \%$ glutaraldehyde in $0.1 \mathrm{~m}$ sodium cacodylate buffer, rinsed, postfixed in $1 \%$ osmium tetroxide and $1 \%$ potassium ferrocyanide, rinsed, en bloc stained in $1 \%$ uranyl acetate, dehydrated with glycol methacrylate, and flat embedded in Epon. The slices were blocked and mounted onto Epon stubs for sectioning parallel to the plane of imaging. Ultrathin sections $(\sim 60 \mathrm{~nm})$ were cut on an ultramicrotome, collected onto Formvar-coated slot grids, and stained with $2 \%$ uranyl acetate and $0.2 \%$ lead citrate. The sections were examined in a JEOL 100CXII transmission electron microscope equipped with a digital camera. Terminals identified in the electron microscope were matched with the fluorescent images on the basis of terminal and target-cell morphology and location.

Flocculectomy. Surgical removal of unilateral flocculus was performed in L7-tau-GFP mice as described previously (Sekirnjak et al., 2003).

Slice preparation and electrophysiology. Animals were deeply anesthetized with Nembutal and decapitated. The hindbrain was quickly removed from the skull and transferred to ice-cold artificial CSF (ACSF) (containing, in mM: $124 \mathrm{NaCl}, 5 \mathrm{KCl}, 1.3 \mathrm{MgSO}_{4}, 26 \mathrm{NaHCO}_{3}, 2.5 \mathrm{CaCl}_{2}$, $1 \mathrm{NaH}_{2} \mathrm{PO}_{4}$, and 11 dextrose), aerated with $95 \% \mathrm{CO}_{2} / 5 \% \mathrm{O}_{2}$. Tissues containing the brainstem, attached with the cerebellum, were dissected and further sectioned in $250 \mu \mathrm{m}$ coronal slices on a Leica VT1000S vibratome in ice-cold aerated ACSF. Slices were transferred to a holding chamber and incubated at $34^{\circ} \mathrm{C}$ for $30 \mathrm{~min}$, followed by RT for $30 \mathrm{~min}$ before recording. Kynurenic acid $(2 \mathrm{mM})$, picrotoxin $(100 \mu \mathrm{M})$, and strychnine $(10 \mu \mathrm{M})$ were added to the ACSF to block glutamatergic, GABAergic, and glycinergic synaptic transmission during recording, respectively.

Micropipettes for whole-cell patch-clamp were prepared by pulling a borosilicate glass pipette (Warner Instruments) with a P-97 puller (Sutter Instruments) to a resistance of 4-8 $\mathrm{M} \Omega$. Internal recording solutions were prepared with the following (in $\mathrm{mm}$ ): $140 \mathrm{~K}$-gluconate, $8 \mathrm{NaCl}, 10$ HEPES, 0.1 EGTA, $2 \mathrm{Mg}$-ATP, and 0.3 Na-GTP. Final pH and osmolarity were adjusted to 7.2 and $280-290$ mOsm, respectively. Biocytin $(0.1 \%)$ (Sigma) was added to the internal solutions for cells to be filled and further processed for immunohistochemistry.

Fluorescence was visualized with a fluorescein filter on an Olympus microscope with a $40 \times$ objective. Neurons were visualized under infra- 

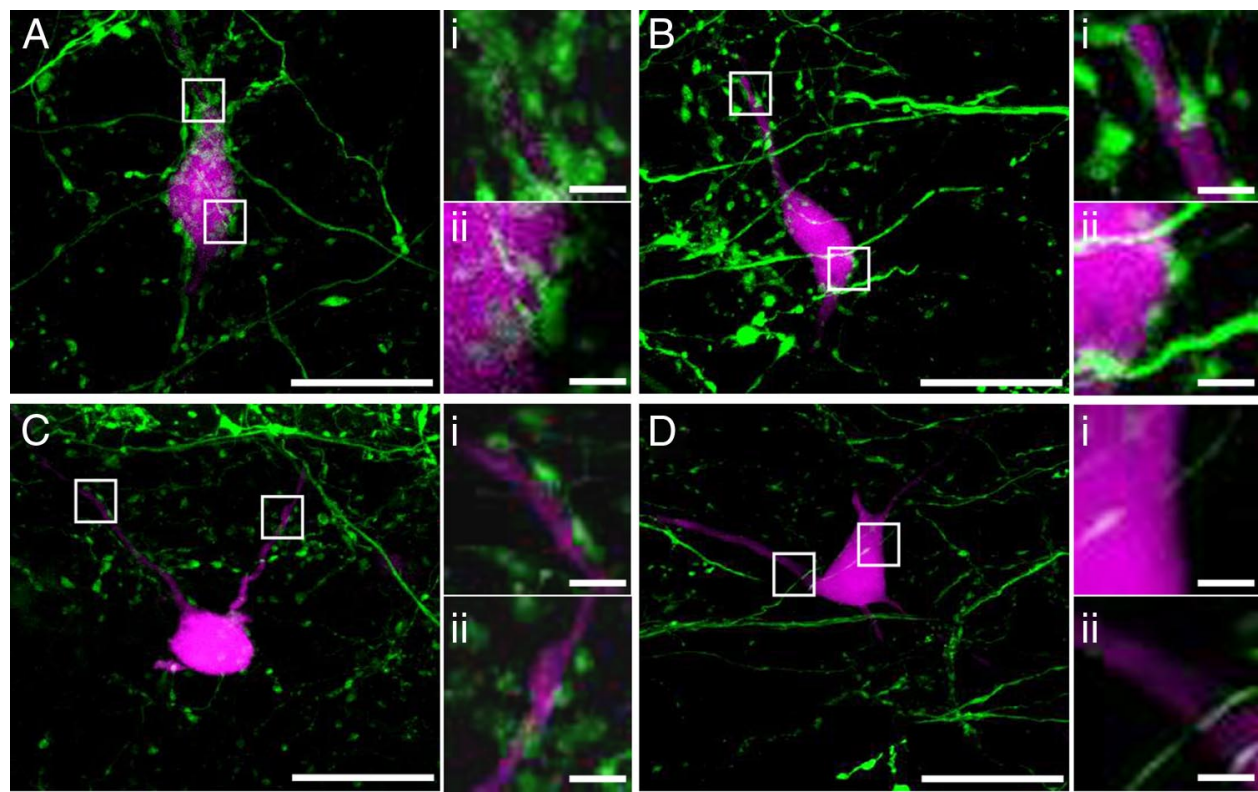

Figure 1. Purkinje cell innervation patterns in the MVN. Neurons in the magnocellular MVN in coronal brainstem slices from L7-tau-GFP mice were filled with biocytin, followed by visualization with streptavidin conjugated with Alexa Fluor 594 to identify different patterns of Purkinje cell terminals. $\boldsymbol{A}$, Example of a neuron with several dozen densely packed Purkinje cell boutons on its soma and proximal dendrites. Ai, Aii, High-magnification view of Purkinje cell contact on the proximal dendrites (Ai) and on the soma (Aii). $\boldsymbol{B}$, Example of a neuron with $<15$ terminals on the soma and proximal dendrites ( $<20 \mu \mathrm{m}$ from soma). $\boldsymbol{B i}$, Bii, High-magnification view of Purkinje cell contact on the proximal dendrites (Bi) and the soma (Bii). $\boldsymbol{C}$, Example of a neuron with Purkinje cell boutons located on the proximal dendritic tree. Ci, Cii, High-magnification view of Purkinje cell contact on the proximal dendrites. $\boldsymbol{D}$, Example of a neuron that is devoid of Purkinje cell boutons. Di, Dii, High-magnification view of soma (Di) and the proximal dendrites (Dii). GFP, green; biocytin-filled cell, magenta. Scale bars: $\boldsymbol{A}-\boldsymbol{D}, 20 \mu \mathrm{m} ; \boldsymbol{i}, \boldsymbol{i i}, 2 \mu \mathrm{m}$.

red illumination with differential interference contrast optics. Wholecell current-clamp recordings were performed with a Multiclamp 700B amplifier. Data were digitized with an ITC-18 board (HEKA) at $40 \mathrm{kHz}$, filtered at $10 \mathrm{kHz}$, and then collected in Macintosh OS X with a customwritten code in Igor Pro 6.

Physiology analysis. Acquisition and analysis of intrinsic firing properties of MVN neurons were performed using Igor Pro 6. All whole-cell recordings were performed with current clamp. Recordings of neurons with $<45 \mathrm{mV}$ spike heights, measured from the threshold to the peak of action potential, were excluded from the group data analysis. To standardize action potential analysis, DC depolarizing or hyperpolarizing current was injected to adjust the cell's spontaneous firing rate to $\sim 10$ $\mathrm{Hz}$; then, action potentials were collected. A total of $5 \mathrm{~s}$ of action potentials were averaged together by aligning them at their peaks. Details on the analysis method were described previously (Bagnall et al., 2007). For measurement of neuronal gain, steps of increasing depolarizing current $(50 \mathrm{pA} / \mathrm{step}, 1 \mathrm{~s})$ were injected until neurons were not able to generate action potential through the entire step, and gains were measured by generating the slope as a best-fit line in the graph of firing rate versus current step amplitude. For postinhibitory rebound analysis, DC depolarizing or hyperpolarizing current was injected to adjust the cell's spontaneous firing rate to $\sim 10 \mathrm{~Hz}$; then, hyperpolarizing current was delivered to hyperpolarize the cells $\sim-30 \mathrm{mV}$ from the average membrane potential of $10 \mathrm{~Hz}$ firing for $1 \mathrm{~s}$. Statistical analyses of physiological parameters were performed using the Mann-Whitney $U$ test. All data points are reported as means \pm SEs.

\section{Results}

Purkinje cells in the cerebellar flocculus synapse on multiple types of vestibular nucleus neurons

The medial vestibular nucleus contains several types of neurons that participate in the VOR, including neurons that are targets of cerebellar Purkinje cells in the floccular lobe (FTNs) and neurons that are presumed to be devoid of Purkinje cell inputs (Ito et al., 1977; du Lac and Lisberger, 1992; Sekirnjak et al., 2003; Ramachandran and Lisberger, 2008). A previous study using L7-tauGFP mice (transgenic mice expressing green fluorescent protein in Purkinje cells) identified a physiologically distinct population of MVN neurons that are densely surrounded by Purkinje cell synaptic boutons originating in the cerebellar flocculus (Sekirnjak et al., 2003). Discrepancies between the small size of this neuronal population ( $\sim 1 \%$ of MVN neurons) and the relatively high rate of encountering FTNs during extracellular recordings in vivo [8-40\% (Sato et al., 1988; du Lac and Lisberger, 1992; Stahl and Simpson, 1995)] along with unpublished observations (M. Bagnall and K. Kolkman) that some MVN neurons appear more sparsely innervated by Purkinje cell terminals prompted a reexamination of the distribution of Purkinje cell synapses onto postsynaptic vestibular nucleus neurons.

To identify neurons that are targeted by Purkinje cell synapses, we made intracellular biocytin fills of MVN neurons in brainstem slices from L7-tau-GFP mice to visualize neuronal somata and dendrites. To maximize the probability of identifying neurons receiving floccular Purkinje cell terminals, we targeted neurons primarily in the magnocellular region of the middle third of the MVN (in the rostrocaudal plane: -5.85 to $-6.21 \mathrm{~mm}$ from bregma; see dotted area in Fig. 2C), where axons and synaptic terminals from floccular Purkinje cells are most dense (Sekirnjak et al., 2003). Ninety-four neurons in this region were randomly selected for dye fills in L7-tau-GFP mice. Of these, the soma and proximal dendrites of 9 neurons were densely surrounded by Purkinje cell terminals (Fig. $1 \mathrm{~A}$ ). Remarkably, of the remaining neurons, 69 (73.4\%) were contacted, albeit sparsely, by Purkinje cell terminals. The majority of these neurons received somatic synapses (2-15 terminals) (Fig. $1 B$ ). Three of the neurons appeared to be innervated by Purkinje cells exclusively on their dendrites (Fig. 1C). Only 16 of 94 neurons (17.0\%) were devoid of somatic or proximal dendritic Purkinje cell terminals (Fig. 1D). Thus, within the magnocellular MVN, most neurons are contacted by Purkinje cell synaptic boutons. 

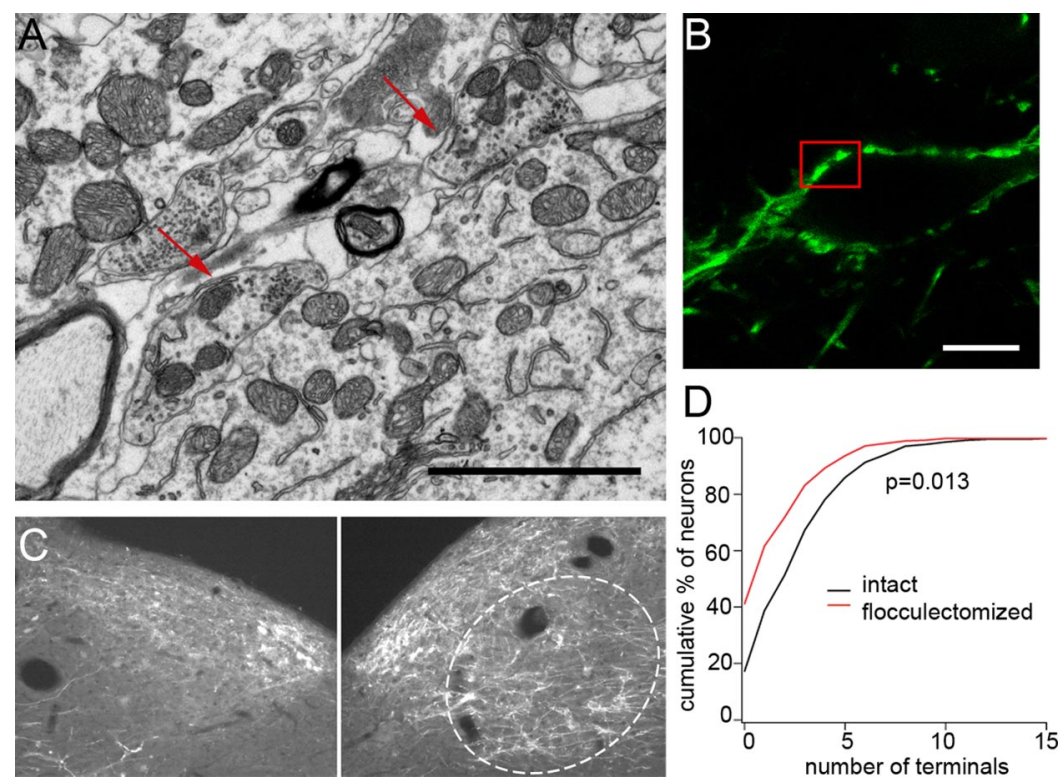

Figure 2. Purkinje cell terminals in the magnocellular MVN originate in the ipsilateral flocculus and form functional synapses. $A$, $B$, Ultrastructure of GFP-positive Purkinje cell terminals was visualized by scanning electron microscopy. $A$, Image of ultrastructure of Purkinje cell terminals surrounding a dFTN. Symmetrical synapses and numerous vesicles (arrows) in the bouton indicate that they form functional synapses. $\boldsymbol{B}$, GFP-positive Purkinje cell terminals surrounding a dFTN. Boxed area was magnified for examining the ultrastructure of synapses shown in A. C, GFP-positive Purkinje cell terminals in the ipsilateral MVN were mostly removed after unilateral surgical ablation of the flocculus (left), while those on the contralateral side of the MVN were intact (dotted area, right). $\boldsymbol{D}$, The numbers of neurons innervated by Purkinje cells were counted on the intact (contralateral) and flocculectomized (ipsilateral) side of the MVN and the data are represented as a cumulative graph. Note the increased numbers of non-FTNs after surgical ablation of the flocculus. Scale bars: $A, 2 \mu \mathrm{m} ; \boldsymbol{B}, 10 \mu \mathrm{m}$.

Neurons in the MVN are predominantly innervated by two subregions of the cerebellum, the nodulus and the flocculus. To determine the origin of GFP-positive axon terminals onto MVN neurons, the flocculus was ablated unilaterally in L7-tau-GFP mice. At 4-6 weeks after unilateral flocculectomy, GFP-positive Purkinje cell terminals were largely absent from the ipsilateral, magnocellular area of the MVN, and no neurons with densely clustered somatic Purkinje cell boutons could be detected (Fig. $2 C)$. This result indicates that Purkinje cell terminals in the magnocellular portion of the MVN originate primarily in the ipsilateral flocculus and confirms that neurons receiving dense Purkinje cell innervation are flocculus target neurons; such neurons will be referred to as dFTNs. In addition, the number of MVN neurons with Purkinje cell terminals on their soma and proximal dendrites was decreased significantly after unilateral flocculectomy. Analysis of the number of Purkinje cell terminals on MVN neurons revealed that flocculectomy increased the number of MVN neurons with no Purkinje cell terminals from 29 of 112 neurons (26\%) to 47 of 110 neurons (43\%). Furthermore, the number of FTNs with 1-3 Purkinje cell terminals on their soma decreased significantly from 68 of 112 neurons (61\%) to 43 of 110 neurons (39\%). This result indicates that Purkinje cell terminals onto neurons located in the magnocellular area of the MVN originate primarily in the ipsilateral flocculus (Fig. 2D). Neurons innervated sparsely by Purkinje cell terminals on the soma and dendrites will be referred to as sFTNs, and neurons devoid of Purkinje cell terminals will be referred to as non-FTNs.

To determine whether Purkinje cell terminals form functional synapses onto FTNs, GFP-positive boutons on the soma or dendrites were identified by fluorescence microscopy, and 12 GFP-positive boutons localized on the soma were examined by serial-section electron microscopy. All GFP-positive boutons contained large numbers $(>100)$ of elongated synaptic vesicles and multiple electron-dense regions (on average, 4.2 electron-dense regions per bouton, range 2-8), which were similar to Purkinje celldeep cerebellar nuclear (DCN) neuron synapses with multiple release sites (Telgkamp et al., 2004), suggesting that they are active, functional synapses. Electrondense regions in the postsynaptic side, forming symmetrical electron-dense regions with its presynaptic side, could be detected in each bouton (Fig. $2 A, B$ ). Additional analysis on 35 GFP-positive boutons localized on the proximal dendrites of FTNs (as far as $50 \mu \mathrm{m}$ away from the soma) by electron microscopy confirmed that these boutons had similar morphological features. These results demonstrate that GFP-positive boutons from floccular Purkinje cells onto FTNs have structural features of functional synapses.

\section{dFTNs are glycinergic neurons}

Cerebellar target neurons in the deep cerebellar nuclei have been traditionally thought to be either glutamatergic neurons projecting to premotor and thalamic nuclei or GABAergic neurons projecting to the inferior olive (Chen and Hillman, 1993). Recently, however, a population of glycinergic neurons in the fastigial nucleus have been identified as Purkinje cell recipient premotor projection neurons (Bagnall et al., 2009; Uusisaari and Knöpfel, 2010). In contrast, the neurotransmitter profile of FTNs has yet to be determined. To examine neurotransmitter expression in FTNs, we immunolabeled Purkinje cell terminals with $\alpha$-calbindin, an intracellular calcium-binding protein, which is highly expressed in Purkinje cells and has been widely used as a marker protein to label Purkinje cells (Bäurle and Grüsser-Cornehls, 1994; for review, see Batini, 1990) and examined Purkinje cell innervation of fluorescent neurons labeled in several different transgenic mouse lines. To evaluate the specificity of calbindin immunoreactivity in Purkinje axon terminals localized in the MVN, coronal sections of L7-tau-GFP mouse brain containing the brainstem attached with the cerebellum were immunolabeled with $\alpha$-calbindin antibody. All GFP-expressing Purkinje cells in the flocculus were colocalized with calbindin immunoreactivity, and all GFP-expressing Purkinje cell terminals densely surrounding MVN neurons were also calbindin positive (Fig. 3). This result confirms the reliability of calbindin immunoreactivity in labeling Purkinje cell terminals on FTNs in the MVN.

Two distinct classes of MVN neurons have been identified using transgenic mouse lines; GIN neurons comprise a subset of local inhibitory GABAergic neurons, and YFP-16 neurons comprise projection neurons, which are glycinergic or glutamatergic (Bagnall et al., 2007; McElvain et al., 2010). To determine whether Purkinje cells innervate projection neurons or local interneurons, we immunolabeled Purkinje cell terminals with $\alpha$-calbindin antibody in coronal sections of the brainstem containing the vestibular nucleus in GIN and YFP-16 mice. More than $95 \%$ of dFTNs identified in YFP-16 mice were colocalized with YFP (animal 1, 155 of 157 FTNs; animal 2, 191 of 201 FTNs) (Fig. 4A). This result is consistent with a prominent influence of 

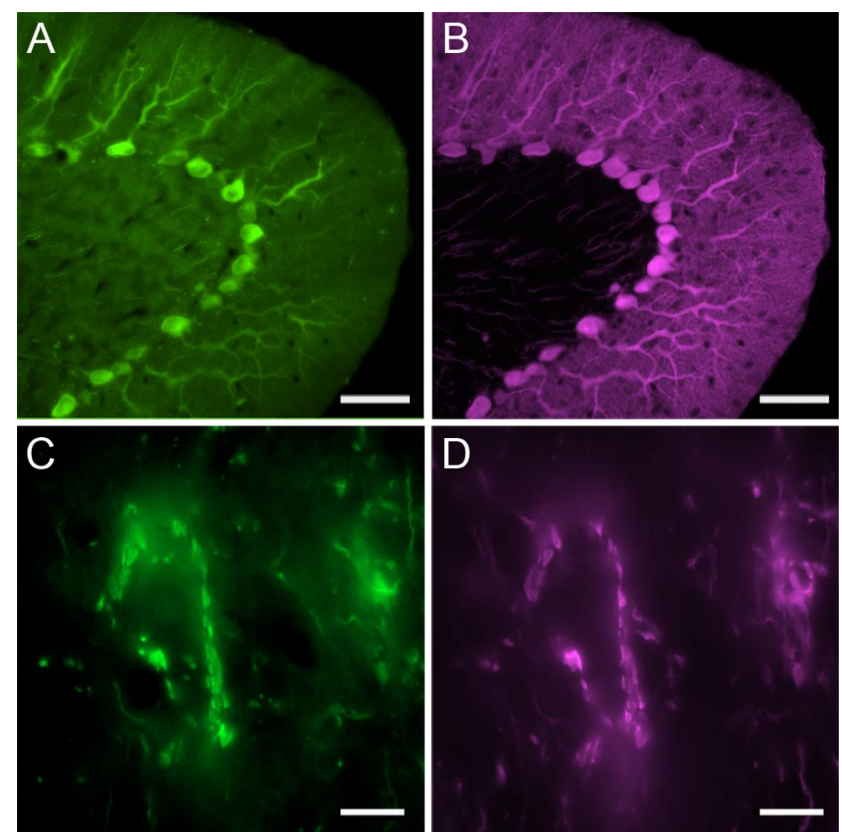

Figure 3. Calbindin is a reliable marker for Purkinje cell terminals in the MVN. $A, B$, GFPpositive Purkinje cells in L7-tau-GFP mice (green) $(\boldsymbol{A})$ were specifically colocalized with the immunoreactivity of $\alpha$-calbindin D-28k antibody (magenta) (B).C, D, GFP-positive Purkinje cell terminals (green) $(\boldsymbol{C})$ were specifically colocalized with the immunoreactivity of $\alpha$-calbindin antibody (magenta) (D) Scale bars: $\boldsymbol{A}, \boldsymbol{B}, 50 \mu \mathrm{m} ; \boldsymbol{C}, \boldsymbol{D}, 10 \mu \mathrm{m}$.

the cerebellum over projection neurons in the MVN ( $\mathrm{du}$ Lac and Lisberger, 1992). In contrast, no dFTNs expressed GFP in GIN mice (Fig. $4 B$ ), suggesting that GABAergic neurons do not receive dense somatic Purkinje cell innervation. The same experimental approach using two other mouse lines expressing GFP in subsets of GABAergic neurons, GAD67-GFP knock-in mice and GAD65 transgenic mice, largely confirmed this result. No dFTNs expressed GFP in the GAD65 line (Fig. 4D), and the majority of neurons expressing GFP in the GAD67-GFP lines (28 of 30) were not dFTNs (Fig. 4C). These results indicate that dFTNs are projection neurons rather than local inhibitory interneurons.

YFP-16 neurons comprise both glycinergic and glutamatergic neurons (Bagnall et al., 2007). To examine whether FTNs are glycinergic, we immunolabeled Purkinje cell terminals with $\alpha$-calbindin antibody in bacterial artificial chromosome (BAC) transgenic mice expressing EGFP under the control of glycine transporter 2 promoter (GlyT2), a useful tool to label glycinergic neurons in the brainstem and cerebellum (Zeilhofer et al., 2005; Bagnall et al., 2009). More than $98 \%$ of dFTNs expressed GFP in the GlyT2 line ( $n=2$; animal 1, 164 of 166 FTNs; animal 2, 226 of 229 FTNs were GlyT2) (Fig. 4E). Together, these results demonstrate that most dFTNs in the MVN are glycinergic projection neurons.

\section{sFTNs include GABAergic and glycinergic neurons}

To examine neurotransmitter expression in sFTNs, Purkinje cell terminals in the MVN were immunolabeled with $\alpha$-calbindin antibody in GIN, GAD65, GAD67-GFP, and GlyT2 mouse brains. GFP-positive neurons localized in the magnocellular area of the MVN in each mouse line were randomly selected, and the numbers of Purkinje cell boutons on the soma and proximal dendrites were counted and analyzed. Twenty-two of 31 (71\%) GlyT2 neurons were sFTNs with 2-15 terminals on the soma and proximal dendrites, while 4 of 31 (13\%) were dFTNs, and 5 of 31
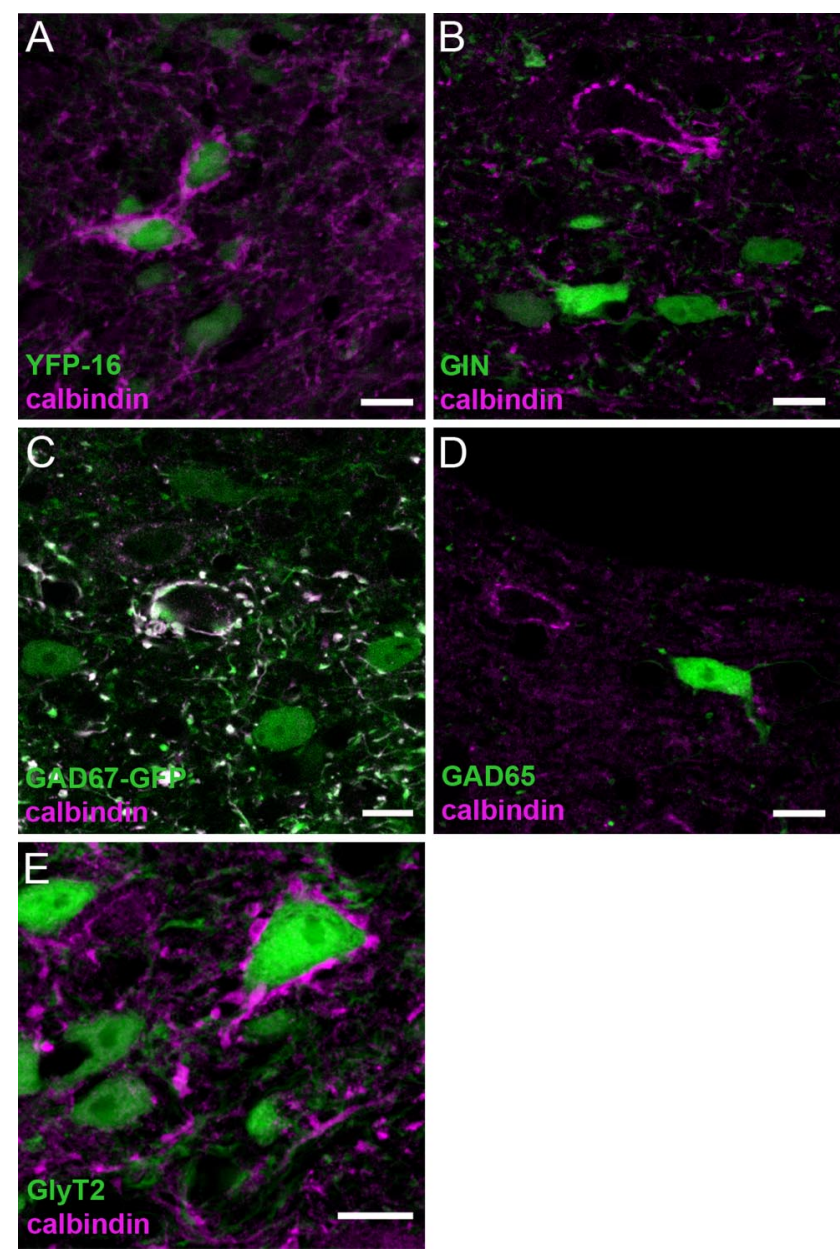

Figure 4. dFTNs in the MVN are exclusively glycinergic. Purkinje cell terminals in $30 \mu \mathrm{m}$ coronal sections of the brainstem from various transgenic mouse lines expressing fluorescent protein (GFP or YFP, green) were immunolabeled with $\alpha$-calbindin antibody (magenta). $A$, YFP-16; $\boldsymbol{B}$, GIN; $\boldsymbol{C}$, GAD67-GFP; $\boldsymbol{D}$, GAD65; $\boldsymbol{E}$, GlyT2. Note that dFTNs could be identified in YFP-16 and GlyT2 lines, but not in other lines, indicating that dFTNs are glycinergic neurons. Scale bar, $20 \mu \mathrm{m}$.

(16\%) were non-FTNs. In GIN mice, 3 of 19 neurons (16\%) were sFTNs, and 16 of 19 neurons (84\%) were non-FTNs. In GAD67GFP mice, 22 of 30 neurons (73\%) were sFTNs, while 2 of 30 neurons (7\%) were dFTNs, and 6 of 30 neurons (20\%) were non-FTNs. No Purkinje cell terminals contacted on GFP-positive neurons in the MVN of GAD65 mice. These results indicate that both glycinergic and GABAergic neurons are sparsely innervated by Purkinje cells, suggesting that sFTNs comprise a greater variety of cell types compared with relatively homogeneous dFTNs.

\section{Intrinsic firing properties of FTNs}

To determine whether excitability of FTNs differs from that of non-FTNs, we measured intrinsic firing properties of FTNs and non-FTNs. Whole-cell patch recording was performed in randomly selected MVN neurons in the magnocellular area of the MVN in L7-tau-GFP mouse $[n=7,>$ p21 (postnatal day 21) and $<\mathrm{p} 32$ ], where the vast majority of floccular Purkinje cell terminals in the MVN are located (Fig. 2C). Neurons were filled with biocytin during the patch-clamp recording, and the number of Purkinje cell terminals was examined after recording. Among 94 neurons recorded, 9 neurons were dFTNs, 69 neurons were sFTNs, and 16 neurons were non-FTNs. All dFTNs (9 of 9) fired spontaneously, whereas $62 \%$ (43 of 69) of sFTNs and $43 \%$ ( 7 of 
Table 1. Intrinsic firing properties of FTNs and non-FTNs

\begin{tabular}{lccc}
\hline & \multicolumn{1}{l}{ dFTNs } & \multicolumn{1}{c}{ sFTNs } & \multicolumn{1}{c}{ non-FTNs } \\
\hline AP width (ms) & $0.80 \pm 0.06$ & $0.90 \pm 0.02$ & $0.82 \pm 0.06$ \\
AHP $(\mathrm{mV})$ & $15.7 \pm 1.5^{*}$ & $20.1 \pm 0.4$ & $20.0 \pm 0.9$ \\
Input resistance $(\mathrm{M} \Omega)$ & $226 \pm 48$ & $181 \pm 16$ & $209 \pm 24$ \\
Resting membrane potential $(\mathrm{mV})$ & $-45.7 \pm 1.2$ & $-42.9 \pm 0.5$ & $-42.1 \pm 0.8$ \\
Maximum firing rate $(\mathrm{Hz})$ & $196 \pm 32$ & $186 \pm 10$ & $209 \pm 24$ \\
Threshold $(\mathrm{mV})$ & $-35.5 \pm 1.9^{*}$ & $-30.6 \pm 0.4$ & $-29.8 \pm 0.8$ \\
Rebound firing $(\mathrm{Hz})$ & $180.9 \pm 22.6^{* *}$ & $17.3 \pm 1.8$ & $16.5 \pm 3.1$ \\
Gain $(\mathrm{Hz} / \mathrm{nA})<80 \mathrm{~Hz}$ & $379 \pm 58^{*}$ & $206 \pm 8.3$ & $186 \pm 16$ \\
\hline
\end{tabular}

All recordings were performed at physiological temperature $\left(34^{\circ} \mathrm{C}\right) . n=9$ (dFTNs), $n=69$ (sFTNs), $n=16$ (non-FTNs). ${ }^{*} p<0.05 ;{ }^{* *} p<0.01$. Values indicate mean \pm SE.

16) of non-FTNs fired spontaneously. Spontaneous firing rates were higher in dFTNs $(28.3 \pm 8.1 \mathrm{~Hz}, p<0.05)$ compared with sFTNs $(12.6 \pm 1.3 \mathrm{~Hz})$ and non-FTNs $(9.8 \pm 2.6 \mathrm{~Hz})$. Measurement of action potential waveform, standardized at a firing rate of $10 \mathrm{~Hz}$ by DC current injection ( -20 to $80 \mathrm{pA}$ ), revealed that dFTNs showed significantly smaller amplitude of afterhyperpolarization compared with sFTNs and non-FTNs. In addition, action potential threshold was significantly lower in dFTNs than sFTNs and non-FTNs. No statistically significant differences in other parameters of intrinsic firing properties were observed (Table 1).

To measure firing properties of FTNs, depolarizing currents of increasing amplitude were applied intracellularly until neurons could not sustain firing during the entire $1 \mathrm{~s}$ of depolarization. Neuronal gain, defined as the slope in current input-firing rate curve, was significantly higher in dFTNs than in either sFTNs or non-FTNs (Table 1, Fig. 5C). Maximum firing rates of sFTNs and non-FTNs varied from $<100$ spikes/s to $>400$ spikes/s, whereas maximum firing rates of dFTNs were confined in relatively small ranges between 120 and 220 spikes/s (Fig. 5D). These results imply that dFTNs are more intrinsically excitable neurons than sFTNs or non-FTNs.

Many DCN neurons generate burst firings after brief hyperpolarization by current injection or synaptic inhibition, and this postinhibitory rebound firing has been suggested as a potential plasticity mechanism in Purkinje cell-DCN neuron synapses (Tadayonnejad et al., 2010; but see Alviña et al., 2008 for opposing point of view). To examine the postinhibitory rebound firing in FTNs, hyperpolarizing current steps were applied to neurons firing at 10 spikes/s (see Materials and Methods). dFTNs showed significantly higher postinhibitory rebound firing rates following $1 \mathrm{~s}$ of hyperpolarization ( $>100$ spikes/s) compared with sFTNs and non-FTNs, in which rebound firing was $<40$ spikes/s (Table 1, Fig. $5 A, B, E)$. Physiological data of dFTNs obtained from the mice older than p21 in this study were consistent with the data from younger mice $(<\mathrm{p} 20)$ reported previously (Sekirnjak et al., 2003). No statistically significant difference in any measured intrinsic firing property was found between sFTNs and non-FTNs (Table 1, Fig. 5). These results indicate that dFTNs are a distinct subset of MVN neurons, distinguished by their dense Purkinje cell innervation pattern and unique intrinsic firing properties. In contrast, sFTNs are a heterogeneous population of MVN neurons that are not physiologically distinguishable from non-FTNs.

\section{Many dFTNs project to ipsilateral ocular motor nuclei}

Electrical stimulation of the flocculus results in ipsiversive eye movements (Sato et al., 1988; du Lac and Lisberger, 1992; Lisberger et al., 1994a), and it has long been hypothesized that a subset of interneurons in the vestibular nucleus (FTNs) are responsible for relaying signals from floccular Purkinje cells to mo- tor neurons in ipsilateral motor nuclei (Ito et al., 1973, 1977). However, no direct evidence supporting this hypothesis has been provided. To identify neurons projecting to the abducens nucleus, rhodamine-conjugated dextrans were unilaterally injected in the abducens of GlyT2 and YFP-16 mice. Retrogradely labeled, abducens-projecting MVN neurons were primarily located in the magnocellular area of the rostral MVN bilaterally, and were largely absent in the caudal MVN (data not shown). In GlyT2 mice, all ipsilateral abducens-projecting neurons, but no contralateral abducens-projecting neurons, were GFP positive, indicating that neurons projecting to the ipsilateral abducens are exclusively glycinergic (Fig. 6A,B). This result is consistent with the previous studies demonstrating that ipsilateral abducensprojecting neurons use glycine as their neurotransmitter (Spencer et al., 1989; Scudder and Fuchs, 1992). Using the same experimental approach in YFP-16 mice, we found that contralateral abducens-projecting neurons were YFP-16 neurons (Fig. $6 C)$. Given that YFP-16 neurons consist of both glycinergic and glutamatergic neurons, this result implies that contralateral abducens-projecting neurons are glutamatergic. Neither ipsilateral nor contralateral abducens-projecting neurons were GFPpositive neurons in GIN mice (data not shown). Thus, MVN neurons are similar to DCN neurons in that glycinergic neurons project to the ipsilateral brainstem, whereas glutamatergic neurons project to the contralateral brainstem (Bagnall et al., 2009).

Because all ipsilateral abducens-projecting neurons are glycinergic, as are most dFTNs, we investigated whether FTNs project to the abducens. Rhodamine-conjugated dextrans were injected into the abducens unilaterally in L7-tau-GFP mice to determine innervation patterns of floccular Purkinje cells on abducens-projecting neurons. Almost half of ipsilateral abducensprojecting neurons were dFTNs $(n=3$; animal 1,36 of $86,41.9 \%$; animal 2, 25 of 60, 41.7\%; animal 3, 48 of 96, 50\% of ipsilateral abducens-projecting neurons were dFTNs), while nearly $36 \%$ of ipsilateral abducens-projecting neurons were sFTNs $(n=3$; animal 1,31 of $86,36.0 \%$; animal 2, 23 of 60 neurons, $38.3 \%$; animal 3,33 of $96,34.4 \%$ of ipsilateral abducens-projecting neurons were dFTNs). Furthermore, $\sim 51 \%$ of total dFTNs were retrogradely labeled from the ipsilateral abducens $(n=3$; animal 1,38 of 69, 55.1\%; animal 2, 25 of 59, 42.4\%; animal 3, 48 of 84, 57.1\% of dFTNs) (Fig. 6D,E). Retrogradely labeling MVN neurons projecting to cerebellum, spinal cord, reticular formation, or contralateral MVN showed that none of these neurons were dFTNs (data not shown). These results demonstrate that most ipsilateral abducens-projecting neurons are either dFTNs or sFTNs.

In contrast to ipsilateral abducens-projecting neurons, no contralateral abducens-projecting neurons were dFTNs. Remarkably, however, more than half of them were identified as sFTNs, receiving 2-10 Purkinje axon terminals on their soma and proximal dendrites $(n=3$; animal 1, 35 of $67,52.2 \%$; animal 2, 17 of $34,50 \%$; animal 3, 30 of 53, 56.6\%) (Fig. $6 F$ ). These results indicate that significant numbers of contralateral abducensprojecting neurons are sFTNs, raising the possibility that Purkinje cells exert influence over eye movements bilaterally.

Horizontal eye movements are mediated by two muscles: the lateral rectus, innervated by the abducens nucleus, and the medial rectus, innervated by the oculomotor nucleus. To examine whether FTNs project to the OMN, neurons in L7-tau-GFP mice were retrogradely labeled by unilateral injection of rhodamineconjugated dextran targeted to the OMN (Fig. 7A). Neurons retrogradely labeled from the ipsilateral $O M N$ were located lateral to the MVN, near the SVN. Several of these OMN-projecting neurons were surrounded by Purkinje cell terminals and thus iden- 
tified as dFTNs (Fig. 7B). Whether sFTNs are among the ipsilateral OMNprojecting neurons could not be determined due to the dense distribution of Purkinje cell axon bundles where these neurons are localized. Retrogradely labeled contralateral OMN-projecting neurons were located in the magnocellular MVN, as reported previously (Sekirnjak and du Lac, 2006; Bergquist et al., 2008) but were largely devoid of Purkinje cell synaptic boutons (Fig. 7C).

\section{Some sFTNs project to the contralateral MVN}

Vestibular neurons crossing the midline and projecting to the contralateral vestibular nucleus (commissural neurons) balance the excitability between two vestibular nuclei and play a critical role in the VOR (Him and Dutia, 2001; Straka et al., 2005; Highstein and Holstein, 2006; Bergquist et al., 2008; Malinvaud et al., 2010). To determine whether floccular Purkinje cells could influence the contralateral MVN through commissural projections, we labeled commissural neurons with BDA in L7-tau-GFP mice and visualized them with streptavidin conjugated with Alexa Fluor 594. Commissurally projecting MVN neurons were located predominantly in the middle third of the rostral-caudal extent of the MVN (data not shown). Z-stack confocal imaging and bouton analysis of randomly selected BDA-labeled neurons in the magnocellular area of the middle MVN revealed that 29 of 61 randomly selected commissural neurons were contacted by $2-10$ Purkinje axon boutons, indicating that they were sFTNs, while 32 of 61 neurons were non-FTNs (Fig. 8). No dFTNs were retrogradely labeled from commissural injections. This result indicates that floccular Purkinje cells not only exert influence on motor nucleusprojecting MVN neurons, but also on neurons that project locally within the MVN.

\section{Discussion}

This study demonstrates that the cerebellum influences a simple sensory-motor behavior via at least five different cell types innervated by Purkinje cells (Fig. 9). Within the circuitry for the horizontal VOR, floccular Purkinje cells target two types of glycinergic and two types of glutamatergic neurons that project axons to ocular motor nuclei. A fifth population of GABAergic neurons provides commissural inputs to the contralateral vestibular complex. Differences in intrinsic physiology, Purkinje cell innervation patterns, and postsynaptic targets imply that the cerebellar control of performance and learning in the VOR is mediated by several distinct microcircuits.

Identification of dFTNs as interneurons mediating cerebellar control of ocular motor nuclei

Our findings that Purkinje cells synapse directly onto premotor neurons in the vestibular nuclei are consistent with classic studies of Ito et al. (1977) who, using anesthetized rabbits, established that electrical stimulation of the flocculus modulated muscle potentials recorded in the medial and lateral rectus muscles (which mediate horizontal eye movements). Two pathways mediating cerebellar control of horizontal eye movements via vestibular nucleus neurons were proposed: an inhibitory pathway onto the ipsilateral abducens nucleus, and an excitatory pathway onto the ipsilateral oculomotor nucleus (Ito et al., 1973). The present study demonstrates that these pathways are mediated by glycinergic and glutamatergic neurons, respectively, and that these neurons receive dense somatic and dendritic Purkinje cell innervation. Although anatomical evidence indicated that glycinergic neurons provide the predominant source of inhibition to the abducens (Spencer and Baker, 1992), our study provides the first direct evidence that FTNs in the medial vestibular nucleus are indeed glycinergic.

\section{Several types of MVN neurons are targeted by Purkinje cell synapses}

In addition to neurons that are densely covered by Purkinje cell synapses, this study revealed that many neurons in the rostral portion of the MVN are sparsely but reliably innervated by Purkinje cell terminals. At least three classes of these sFTNs are in a position to influence the horizontal VOR via distinct pathways to the abducens nucleus and to the contralateral vestibular nucleus 

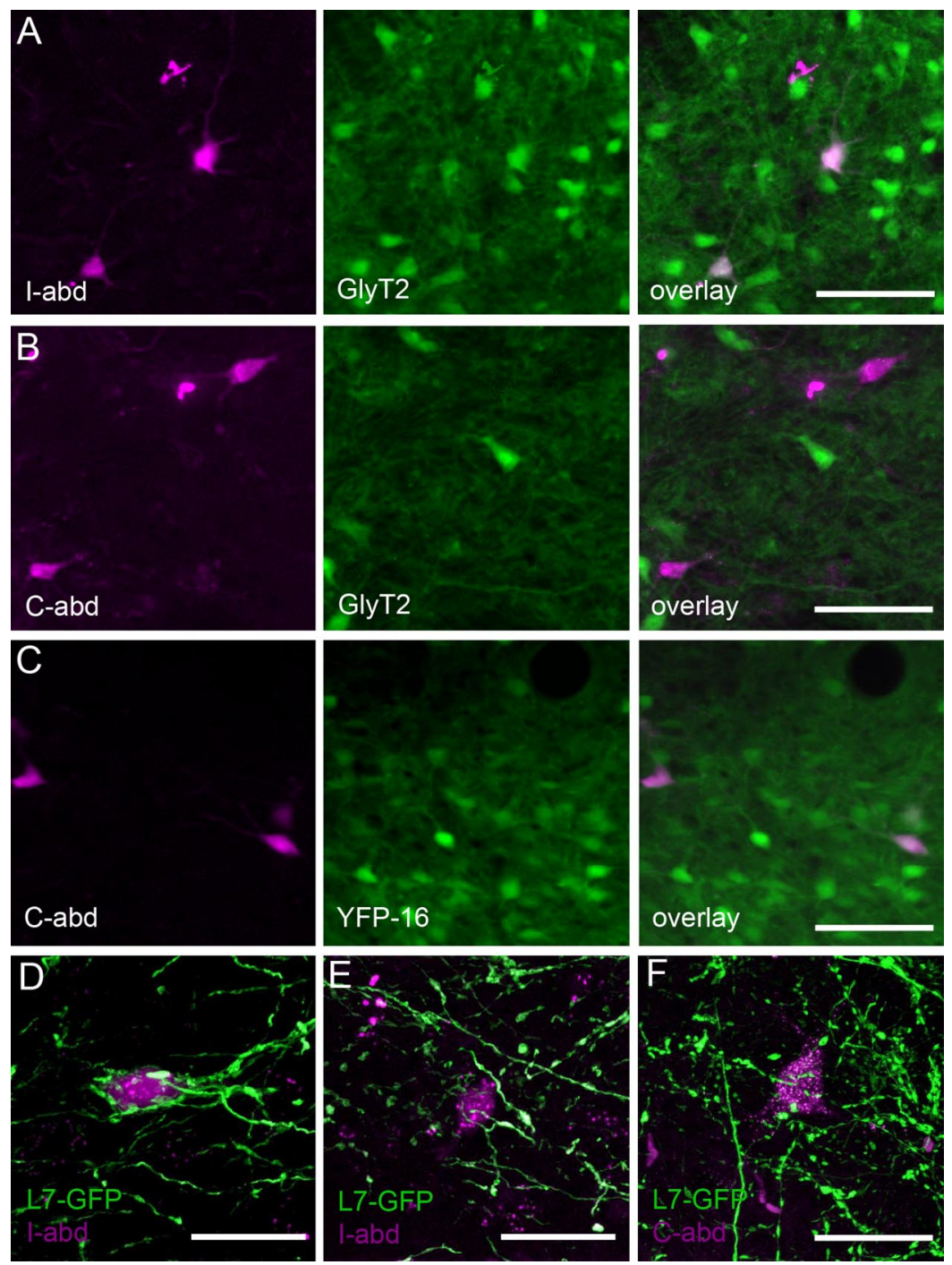

Figure 6. FTNs project bilaterally to the abducens nucleus. Abducens-projecting neurons were retrogradely labeled with dextran conjugated with rhodamine (magenta) in transgenic mice expressing fluorescence proteins under various promotors. $A, B$, Retrograde labeling of abducens-projecting neurons in GlyT2 mouse. $\boldsymbol{A}$, Ipsilateral abducens-projecting neurons are all GFP positive in GlyT2 mice. $\boldsymbol{B}$, None of the contralateral abducens-projecting neurons are GlyT2 positive. $C$, Retrograde labeling of abducens-projecting neurons in YFP16. Contralateral abducens-projecting neurons are YFP positive in YFP-16 mice. $\boldsymbol{D}-\boldsymbol{F}$, Retrograde labeling of abducens-projecting neurons in L7-tau-GFP mice. D, Example of dFTNs among ipsilateral abducens-projecting neurons. $\boldsymbol{E}$, Example of sFTNs among ipsilateral abducensprojecting neurons. $\boldsymbol{F}$, Example of sFTNs among contralateral abducens-projecting neurons. Scale bars: $\boldsymbol{A}-\boldsymbol{C}, 50 \mu \mathrm{m} ; \boldsymbol{D}-\boldsymbol{F}, 20 \mu \mathrm{m}$.
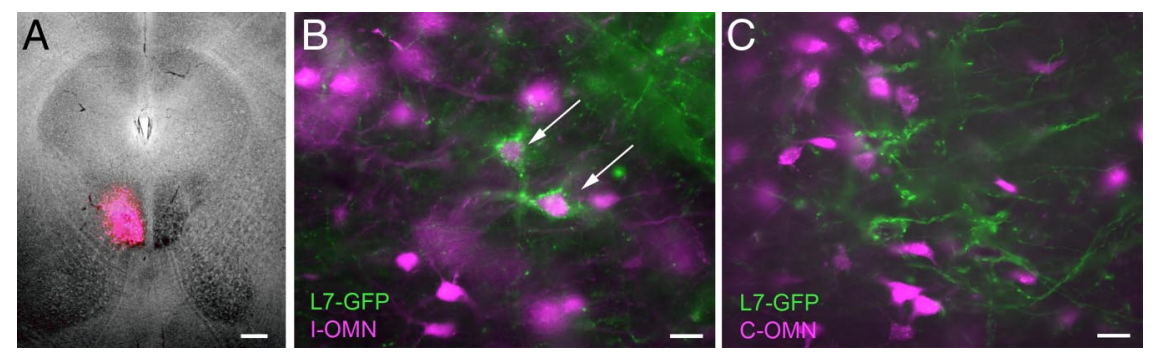

Figure 7. dFTNs in the lateral vestibular complex project to the ipsilateral oculomotor nucleus. $\boldsymbol{A}, 0 \mathrm{MN}$-projecting neurons were retrogradely labeled with rhodamine-conjugated dextran (magenta) in L7-tau-GFP mice (green). $\boldsymbol{B}$, Some ipsilateral 0MN-projecting neurons are dFTNs (arrows). C, No contralateral 0MN-projecting neurons were dFTNs. Scale bars: $\boldsymbol{A}, 200 \mu \mathrm{m} ; \boldsymbol{B}, \boldsymbol{C}, 25 \mu \mathrm{m}$.

(Fig. 9). Purkinje cell inhibition of glycinergic sFTNs and dFTNs innervating the ipsilateral abducens nucleus would facilitate ipsiversive eye movements, as would inhibition of glutamatergic dFTNs innervating the ipsilateral OMN. Similarly, Purkinje cell inhibition of contralateral glutamatergic sFTNs that innervate the abducens would promote ipsiversive eye movements. Insights into the role of sFTNs that project to the contralateral MVN will require identification of the cell types that they innervate.

Examination of intrinsic excitability indicates that ion channel expression and/or regulation is likely to differ between FTNs that are densely versus sparsely innervated by Purkinje cells. dFTNs are a relatively homogenous population of fast-firing neurons with exceptionally strong postinhibitory rebound firing, whereas sFTNs are heterogeneous with respect to spike width, excitability, and postinhibitory rebound firing. It is interesting that the two classes of glycinergic neurons projecting to the ipsilateral abducens nucleus differ both physiologically and with respect to Purkinje cell innervation density. From a developmental perspective, this observation implies that Purkinje cell axons either specifically recognize and target for dense innervation a small subset of neurons, or, alternatively, that Purkinje cells initially contact random MVN neurons and subsequently refine synapse number and postsynaptic ion channel expression in an activity-dependent manner.

The heterogeneity of neurons mediating cerebellar influence over the VOR described in this study was anticipated by scattered reports in the literature indicating that FTNs comprise multiple cell types with respect to synaptic inputs, neurotransmitter expression, and firing responses during head and eye movements. Although all FTNs respond to peripheral vestibular stimulation, only a subset are monosynaptically excited from the vestibular nerve, while many are disynaptically or polysynaptically activated (du Lac and Lisberger, 1992; Lisberger et al., 1994b; Partsalis et al., 1995). Electron micrographic analyses indicate that Purkinje cell synapses contact GABAergic, glycinergic, and presumed glutamatergic neurons in the vestibular nucleus (De Zeeuw and Berrebi, 1996). In behaving animals, the firing responses of FTNs during ipsiversive versus contraversive eye movements vary widely (Lisberger et al., 1994a). Although postinhibitory rebound in FTNs has not been quantified in vivo, exemplar responses reported in several species indicate that some FTNs exhibit substantial rebound firing following cerebellar inhibition, while others show little or no rebound firing (du Lac and Lisberger, 1992; Lisberger et al., 1994b; Stahl and Simpson, 1995). Together, these findings indicate that FTNs comprise a physiologically and functionally diverse population. 

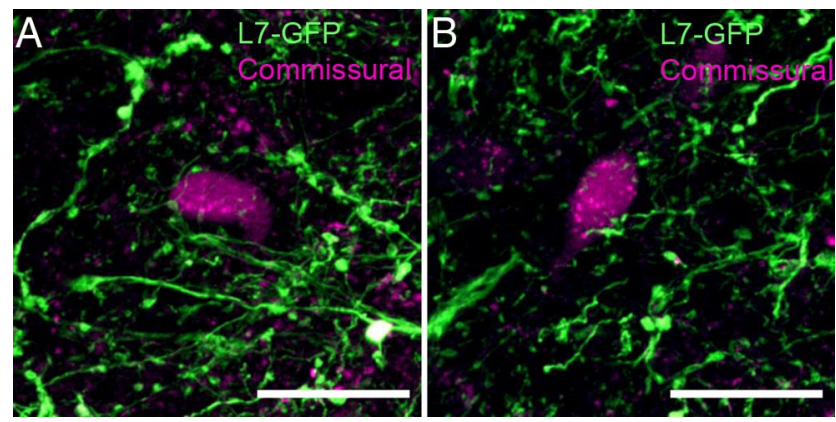

Figure 8. Some commissural MVN neurons are sFTNs. Commissural MVN neurons were retrogradely labeled with biotin-conjugated dextran (magenta) in L7-tau-GFP mouse (green). $\boldsymbol{A}$, $\boldsymbol{B}$, Examples of sFTNs among commissural neurons located in the caudal MVN $(\boldsymbol{A})$ and rostral MVN (B). Scale bars, $20 \mu \mathrm{m}$.

Given the criteria for identifying FTNs in vivo, it is likely that neurons receiving sparse somatic Purkinje cell innervation were overlooked in previous recordings, which defined FTNs by a complete cessation of firing at monosynaptic latencies following a single electrical shock to the flocculus (Sato et al., 1988; du Lac and Lisberger, 1992; Lisberger et al., 1994a; Stahl and Simpson, 1995; Zhang et al., 1995; Babalian and Vidal, 2000). Given the endogenous pacemaking capabilities of MVN neurons (Lin and Carpenter, 1993; Gittis and du Lac, 2007) and their ongoing excitation by vestibular inputs, the cessation of firing by cerebellar stimulation would require strong hyperpolarization of the cell body. That is, the ongoing firing of densely innervated FTNs should be much more strongly affected by Purkinje cell inhibition than that of sparsely innervated FTNs. How might cerebellar activity influence signaling in sFTNs? The dendritic distribution of cerebellar synapses suggests the intriguing possibility that Purkinje cells modulate synaptic transmission from vestibular nerve synapses onto the dendrites of FTNs while having relatively little influence on somatically generated firing. This scenario would provide an explanation for the striking, unexplained observation that eye movements evoked by electrical stimulation of the vestibular apparatus are reduced by $90 \%$ with a single, appropriately timed stimulus to the flocculus (du Lac and Lisberger, 1992).

Functional significance of cerebellar influence over multiple microcircuits subserving the VOR

Our findings necessitate a revision of the traditional view of VOR circuitry in which two functionally distinct types of sensorymotor interneurons have been identified: PVPs (position vestibular pause interneurons), which excite abducens motoneurons, are devoid of Purkinje cell influence, and are not modified during VOR learning, and FTNs, which mediate cerebellar influence over VOR performance and adaptive plasticity (Lisberger and Pavelko, 1988; Scudder and Fuchs, 1992; Ramachandran and Lisberger, 2008). The combined anatomical, molecular, and cellular physiological analyses presented in this study indicate that several types of FTNs project to the abducens and that some FTNs are glutamatergic (Fig. 6, 7). The finding that some neurons retrogradely labeled from the contralateral abducens were consistently devoid of Purkinje cell terminals is consistent with the notion of a fast pathway that contributes to driving the VOR but that is not modified by the cerebellum (Lisberger and Pavelko, 1988; Ramachandran and Lisberger, 2008). Although it is conceivable that these "unmodifiable pathway" neurons receive undetected Pur-

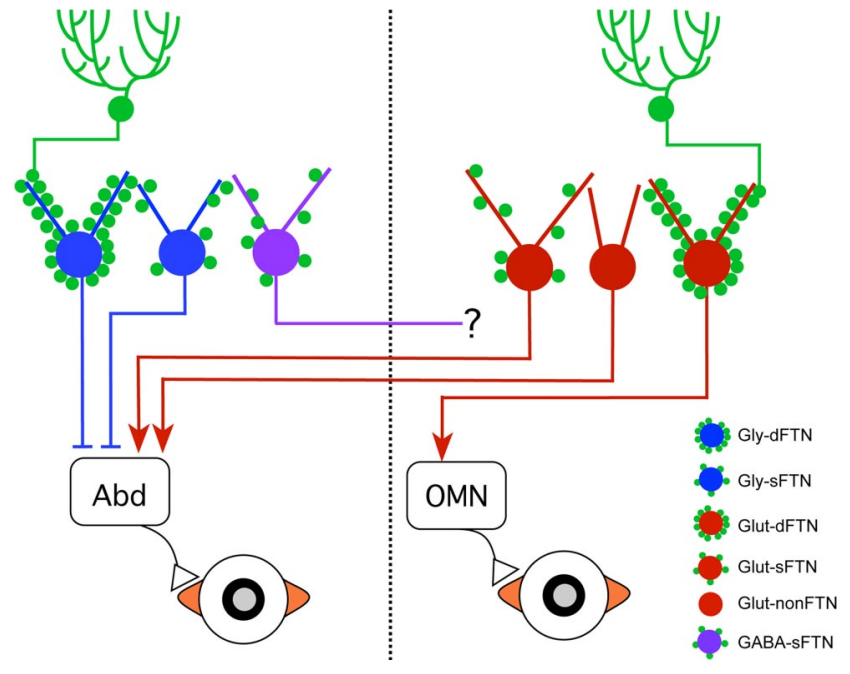

Figure 9. Schematic diagram of cerebellar control of horizontal eye movement. Three types ofFTNs project to the abducens nucleus: densely and sparsely innervated glycinergic (blue) FTNs project ipsilaterally (leftmost and adjacent neurons), while sparsely innervated glutamatergic (red) neurons project contralaterally (neuron on far right). GABAergic neurons (purple) project axons across the midline to contralateral MVN.

kinje cell synapses on distal dendrites, direct vestibular nerve inputs onto their somata would not be affected by cerebellar activity. Our results indicate that in addition to an unmodifiable pathway, the VOR circuit comprises several microcircuits that may be differentially modified by the cerebellum.

\section{Implications for other cerebellar nuclei}

Extensive Purkinje cell innervation of constituent neurons establishes the vestibular nuclei as bona fide cerebellar output nuclei that differ from the actual deep cerebellar nuclei, primarily in their position ventral to (rather than dorsal to) the fourth ventricle and in their relatively direct connections with sensory inputs and motor outputs. This study demonstrates several parallels at the cellular and circuit levels between neurons in vestibular and cerebellar nuclei. Neurons with rapid action potentials and firing capacities and that project axons to contralateral motor, premotor, or thalamic nuclei are exclusively glutamatergic, while similar neurons in the lateral cerebellar and vestibular nuclei that project axons ipsilaterally are glycinergic (Bagnall et al., 2009; Uusisaari and Knöpfel, 2010). While many projection neurons exhibit pronounced postinhibitory rebound firing, the magnitude and time course vary considerably (Uusisaari et al., 2007; Rowland and Jaeger, 2008; Tadayonnejad et al., 2010). Additional targets of Purkinje cell synapses include GABAergic neurons that project to the inferior olive and have wider action potentials than premotor projection neurons, and small glycinergic neurons, which tend to receive Purkinje cell synapses on their dendrites (Uusisaari and Knöpfel, 2010). The similarities between cerebellar output nuclei suggest that the diversity of microrcircuits dedicated to a single behavior that we have established for the vestibular system are likely to extend to other behaviors that are modified by the cerebellum.

Several behavioral paradigms have served as models for investigating the circuit and cellular substrates of cerebellar learning, including classical conditioning of eyelid responses saccadic adaptation, and motor learning in the VOR (for review, see Christian and Thompson, 2003; Iwamoto and Kaku, 2010; du Lac et al., 1995, respectively). Increasing evidence implicates cerebellar output nuclei in learning and memory storage (Kassardjian et al., 
2005; Ohyama et al., 2006; Shutoh et al., 2006). Intriguingly, a number of candidate cellular mechanisms of plasticity have been identified in cerebellar and vestibular nuclei (Zhang and Linden, 2006; McElvain et al., 2010; Zheng and Raman, 2010). Examining and manipulating the roles of specific FTNs in VOR adaptation should reveal general principles about the modular control of cerebellar learning.

\section{References}

Aizenman CD, Linden DJ (2000) Rapid, synaptically driven increases in the intrinsic excitability of cerebellar deep nuclear neurons. Nat Neurosci 3:109-111.

Albus JS (1971) A theory of cerebellar function. Math Biosci 10:25-61.

Alviña K, Walter JT, Kohn A, Ellis-Davies G, Khodakhah K (2008) Questioning the role of rebound firing in the cerebellum. Nat Neurosci 11:1256-1258.

Apps R, Garwicz M (2005) Anatomical and physiological foundations of cerebellar information processing. Nat Rev Neurosci 6:297-311.

Babalian AL, Vidal PP (2000) Floccular modulation of vestibuloocular pathways and cerebellum-related plasticity: an in vitro whole brain study. J Neurophysiol 84:2514-2528.

Bagnall MW, Stevens RJ, du Lac S (2007) Transgenic mouse lines subdivide medial vestibular nucleus neurons into discrete, neurochemically distinct populations. J Neurosci 27:2318-2330.

Bagnall MW, Zingg B, Sakatos A, Moghadam SH, Zeilhofer HU, du Lac S (2009) Glycinergic projection neurons of the cerebellum. J Neurosci 29:10104-10110.

Batini C (1990) Cerebellar localization and colocalization of GABA and calcium binding protein-D28K. Arch Ital Biol 128:127-149.

Bäurle J, Grüsser-Cornehls U (1994) Calbindin D-28k in the lateral vestibular nucleus of mutant mice as a tool to reveal Purkinje cell plasticity. Neurosci Lett 167:85-88.

Bergquist F, Ludwig M, Dutia MB (2008) Role of the commissural inhibitory system in vestibular compensation in the rat. J Physiol 586:4441-4452.

Chen S, Hillman DE (1993) Colocalization of neurotransmitters in the deep cerebellar nuclei. J Neurocytol 22:81-91.

Christian KM, Thompson RF (2003) Neural substrates of eyeblink conditioning: acquisition and retention. Learn Mem 10:427-455.

De Zeeuw CI, Berrebi AS (1996) Individual Purkinje cell axons terminate on both inhibitory and excitatory neurons in the cerebellar and vestibular nuclei. Ann N Y Acad Sci 781:607-610.

De Zeeuw CI, Yeo CH (2005) Time and tide in cerebellar memory formation. Curr Opin Neurobiol 15:667-674.

De Zeeuw CI, Hansel C, Bian F, Koekkoek SK, van Alphen AM, Linden DJ, Oberdick J (1998) Expression of a protein kinase C inhibitor in Purkinje cells blocks cerebellar LTD and adaptation of the vestibulo-ocular reflex. Neuron 20:495-508.

du Lac S, Lisberger SG (1992) Eye movements and brainstem neuronal responses evoked by cerebellar and vestibular stimulation in chicks. J Comp Physiol A 171:629-638.

du Lac S, Raymond JL, Sejnowski TJ, Lisberger SG (1995) Learning and memory in the vestibulo-ocular reflex. Annu Rev Neurosci 18:409-441.

Feng G, Mellor RH, Bernstein M, Keller-Peck C, Nguyen QT, Wallace M, Nerbonne JM, Lichtman JW, Sanes JR (2000) Imaging neuronal subsets in transgenic mice expressing multiple spectral variants of GFP. Neuron 28:41-51.

Gittis AH, du Lac S (2006) Intrinsic and synaptic plasticity in the vestibular system. Curr Opin Neurobiol 16:385-390.

Gittis AH, du Lac S (2007) Firing properties of GABAergic versus nonGABAergic vestibular nucleus neurons conferred by a differential balance of potassium currents. J Neurophysiol 97:3986-3996.

Highstein SM, Holstein GR (2006) The anatomy of the vestibular nuclei. Prog Brain Res 151:157-203.

Him A, Dutia MB (2001) Intrinsic excitability changes in vestibular nucleus neurons after unilateral deafferentation. Brain Res 908:58-66.

Ito M (1972) Neural design of the cerebellar motor control system. Brain Res 40:81-84.

Ito M, Nisimaru N, Yamamoto M (1973) Specific neural connections for the cerebellar control of vestibulo-ocular reflexes. Brain Res 60:238-243.

Ito M, Nisimaru N, Yamamoto M (1977) Specific patterns of neuronal con- nexions involved in the control of the rabbit's vestibulo-ocular reflexes by the cerebellar flocculus. J Physiol 265:833-854.

Iwamoto Y, Kaku Y (2010) Saccade adaptation as a model of learning in voluntary movements. Exp Brain Res 204:145-162.

Kassardjian CD, Tan YF, Chung JY, Heskin R, Peterson MJ, Broussard DM (2005) The site of a motor memory shifts with consolidation. J Neurosci 25:7979-7985.

Lin Y, Carpenter DO (1993) Medial vestibular neurons are endogenous pacemakers whose discharge is modulated by neurotransmitters. Cell Mol Neurobiol 13:601-613.

Lisberger SG, Pavelko TA (1988) Brain stem neurons in modified pathways for motor learning in the primate vestibulo-ocular reflex. Science 242:771-773.

Lisberger SG, Pavelko TA, Broussard DM (1994a) Responses during eye movements of brain stem neurons that receive monosynaptic inhibition from the flocculus and ventral paraflocculus in monkeys. J Neurophysiol 72:909-927.

Lisberger SG, Pavelko TA, Broussard DM (1994b) Neural basis for motor learning in the vestibuloocular reflex of primates. I. Changes in the responses of brain stem neurons. J Neurophysiol 72:928-953.

López-Bendito G, Sturgess K, Erdélyi F, Szabó G, Molnár Z, Paulsen O (2004) Preferential origin and layer destination of GAD65-GFP cortical interneurons. Cereb Cortex 14:1122-1133.

Malinvaud D, Vassias I, Reichenberger I, Rössert C, Straka H (2010) Functional organization of vestibular commissural connections in frog. J Neurosci 30:3310-3325.

Marr D (1969) A theory of cerebellar cortex. J Physiol 202:437-470.

Mauk MD, Garcia KS, Medina JF, Steele PM (1998) Does cerebellar LTD mediate motor learning? Toward a resolution without a smoking gun. Neuron 20:359-362.

McElvain LE, Bagnall MW, Sakatos A, du Lac S (2010) Bidirectional plasticity gated by hyperpolarization controls the gain of postsynaptic firing responses at central vestibular nerve synapses. Neuron 68:763-775.

Medina JF, Nores WL, Ohyama T, Mauk MD (2000) Mechanisms of cerebellar learning suggested by eyelid conditioning. Curr Opin Neurobiol 10:717-724

Nelson AB, Gittis AH, du Lac S (2005) Decreases in CaMKII activity trigger persistent potentiation of intrinsic excitability in spontaneously firing vestibular nucleus neurons. Neuron 46:623-631.

Ohyama T, Nores WL, Medina JF, Riusech FA, Mauk MD (2006) Learninginduced plasticity in deep cerebellar nucleus. J Neurosci 26:12656-12663.

Oliva AA Jr, Jiang M, Lam T, Smith KL, Swann JW (2000) Novel hippocampal interneuronal subtypes identified using transgenic mice that express green fluorescent protein in GABAergic interneurons. J Neurosci 20:3354-3368.

Partsalis AM, Zhang Y, Highstein SM (1995) Dorsal Y group in the squirrel monkey. I. Neuronal responses during rapid and long-term modifications of the vertical VOR. J Neurophysiol 73:615-631.

Person AL, Raman IM (2010) Deactivation of L-type Ca current by inhibition controls LTP at excitatory synapses in the cerebellar nuclei. Neuron 66:550-559.

Pugh JR, Raman IM (2006) Potentiation of mossy fiber EPSCs in the cerebellar nuclei by NMDA receptor activation followed by postinhibitory rebound current. Neuron 51:113-123.

Ramachandran R, Lisberger SG (2008) Neural substrate of modified and unmodified pathways for learning in monkey vestibuloocular reflex. J Neurophysiol 100:1868-1878.

Rowland NC, Jaeger D (2008) Responses to tactile stimulation in deep cerebellar nucleus neurons result from recurrent activation in multiple pathways. J Neurophysiol 99:704-717.

Ruigrok TJ (2010) Ins and outs of cerebellar modules. Cerebellum. Advance online publication. Retrieved Mar. 16, 2010. doi:10.1007/s12311010-0164-y.

Sato Y, Kanda K, Kawasaki T (1988) Target neurons of floccular middle zone inhibition in medial vestibular nucleus. Brain Res 446:225-235.

Scudder CA, Fuchs AF (1992) Physiological and behavioral identification of vestibular nucleus neurons mediating the horizontal vestibuloocular reflex in trained rhesus monkeys. J Neurophysiol 68:244-264.

Sekirnjak C, du Lac S (2006) Physiological and anatomical properties of mouse medial vestibular nucleus neurons projecting to the oculomotor nucleus. J Neurophysiol 95:3012-3023.

Sekirnjak C, Vissel B, Bollinger J, Faulstich M, du Lac S (2003) Purkinje cell 
synapses target physiologically unique brainstem neurons. J Neurosci 23:6392-6398.

Shutoh F, Katoh A, Ohki M, Itohara S, Tonegawa S, Nagao S (2003) Role of protein kinase $\mathrm{C}$ family in the cerebellum-dependent adaptive learning of horizontal optokinetic response eye movements in mice. Eur J Neurosci $18: 134-142$.

Shutoh F, Ohki M, Kitazawa H, Itohara S, Nagao S (2006) Memory trace of motor learning shifts transsynaptically from cerebellar cortex to nuclei for consolidation. Neuroscience 139:767-777.

Spencer RF, Baker R (1992) GABA and glycine as inhibitory neurotransmitters in the vestibuloocular reflex. Ann N Y Acad Sci 656:602-611.

Spencer RF, Wenthold RJ, Baker R (1989) Evidence for glycine as an inhibitory neurotransmitter of vestibular, reticular, and prepositus hypoglossi neurons that project to the cat abducens nucleus. J Neurosci 9:2718-2736.

Stahl JS, Simpson JI (1995) Dynamics of rabbit vestibular nucleus neurons and the influence of the flocculus. J Neurophysiol 73:1396-1413.

Straka H, Vibert N, Vidal PP, Moore LE, Dutia MB (2005) Intrinsic membrane properties of vertebrate vestibular neurons: function, development and plasticity. Prog Neurobiol 76:349-392.

Tadayonnejad R, Anderson D, Molineux ML, Mehaffey WH, Jayasuriya K, Turner RW (2010) Rebound discharge in deep cerebellar nuclear neurons in vitro. Cerebellum 9:352-374.

Tamamaki N, Yanagawa Y, Tomioka R, Miyazaki J, Obata K, Kaneko T (2003) Green fluorescent protein expression and colocalization with cal- retinin, parvalbumin, and somatostatin in the GAD67-GFP knock-in mouse. J Comp Neurol 467:60-79.

Telgkamp P, Padgett DE, Ledoux VA, Woolley CS, Raman IM (2004) Maintenance of high-frequency transmission at Purkinje to cerebellar nuclear synapses by spillover from boutons with multiple release sites. Neuron 41:113-126.

Uusisaari M, Knöpfel T (2010) Functional classification of neurons in the mouse lateral cerebellar nuclei. Cerebellum. Advance online publication. Retrieved Nov. 10, 2010. doi:10.1007/s12311-010-0240-3.

Uusisaari M, Obata K, Knöpfel T (2007) Morphological and electrophysiological properties of GABAergic and non-GABAergic cells in the deep cerebellar nuclei. J Neurophysiol 97:901-911.

Voogd J, Glickstein M (1998) The anatomy of the cerebellum. Trends Cogn Sci 2:307-313.

Zeilhofer HU, Studler B, Arabadzisz D, Schweizer C, Ahmadi S, Layh B, Bösl MR, Fritschy JM (2005) Glycinergic neurons expressing enhanced green fluorescent protein in bacterial artificial chromosome transgenic mice. J Comp Neurol 482:123-141.

Zhang W, Linden DJ (2006) Long-term depression at the mossy fiber-deep cerebellar nucleus synapse. J Neurosci 26:6935-6944.

Zhang Y, Partsalis AM, Highstein SM (1995) Properties of superior vestibular nucleus flocculus target neurons in the squirrel monkey. I. General properties in comparison with flocculus projecting neurons. J Neurophysiol 73:2261-2278.

Zheng N, Raman IM (2010) Synaptic inhibition, excitation, and plasticity in neurons of the cerebellar nuclei. Cerebellum 9:56-66. 